\title{
MATRIX KERNELS FOR THE GAUSSIAN ORTHOGONAL AND SYMPLECTIC ENSEMBLES
}

\author{
by Craig A. TRACY \& Harold WIDOM
}

\section{Introduction.}

For a large class of finite $N$ determinantal processes the limiting distribution, as $N \rightarrow \infty$, of the right-most "particle" is expressible as the Fredholm determinant of an operator $K_{\text {Airy }}$ with kernel

$$
\frac{A(x) A^{\prime}(y)-A^{\prime}(x) A(y)}{x-y}=\int_{0}^{\infty} A(x+z) A(y+z) d z,
$$

where $A(x)=\operatorname{Ai}(x)$ is the Airy function. (See [5], [11] for recent reviews.) Typically these results are proved by first establishing that for finite $N$ the distribution of the right-most particle is the Fredholm determinant of an operator $K_{N}$. The limit theorem then follows once one proves $K_{N} \rightarrow K_{\text {Airy }}$ in trace norm. The classic example is the finite $N$ Gaussian Unitary Ensemble (GUE) where $K_{N}$ is the Hermite kernel [6] and the limit $N \rightarrow \infty$ is the edge scaling limit of the largest eigenvalue. In this case the unitary invariance of the underlying probability measure is manifest.

As is well known one can also consider the edge scaling of the largest eigenvalue for the Gaussian Orthogonal Ensemble (GOE) or the Gaussian Symplectic Ensemble (GSE) [9]. These limiting distributions are now widely believed to describe the universal behavior of a right-most particle for a large class of processes under additional symmetry restrictions [3], [8]. In

Keywords: random matrices, Gaussian orthogonal, symplectic ensembles. Math. classification: 60F99, 47B34. 
the orthogonal and symplectic ensembles, the distribution of the largest eigenvalue is the square root of a Fredholm determinant of an operator $K_{N}$ whose kernel is now a $2 \times 2$ matrix kernel (of course, different $K_{N}$ for different ensembles). In [9] the derivation of the limiting distributions for the largest eigenvalue did not use limiting kernels, as it did for the ensembles scaled in the bulk. Instead, differential equations determining the the finite $N$ distributions were obtained first and then a limiting argument was applied to these.

The edge-scaled kernels are already in the literature [2], [1], but as far as we know limit theorems in the operator norms that give convergence of the determinants are not. Because of the current interest in these ensembles, it seems useful to state and prove such theorems for the matrix kernels. For GSE we shall establish convergence in trace norm to a limiting operator $K_{\mathrm{GSE}}$. The GOE case is more awkward and for that we shall use weighted Hilbert spaces and an extension of the determinant involving the regularized 2-determinant, and convergence to $K_{\mathrm{GOE}}$ will be in a combination of Hilbert-Schmidt and trace norms. These will give convergence of the determinants.

\section{The finite $N$ kernels.}

We shall follow the notation of [10], more or less. For both finite $N$ ensembles we denote by $K_{N}(x, y)$ the matrix kernel (and $K_{N}$ the corresponding operator) such that the expected value of $\prod_{k}\left(1+f\left(\lambda_{k}\right)\right)$ is given by

$$
E\left(\prod_{k}\left(1+f\left(\lambda_{k}\right)\right)\right)=\sqrt{\operatorname{det}\left(I+K_{N} f\right)} .
$$

Here $f$ is a "general" function and in the determinant it denotes multiplication by $f$. In particular the probability of finding no eigenvalues in a set $J$ is given by

$$
\sqrt{\operatorname{det}\left(I-K_{N} \chi_{J}\right)}
$$

Here are the formulas for $K_{N}$. We set $\varepsilon(x)=\frac{1}{2} \operatorname{sgn} x$, denote by $\varepsilon$ the operator with kernel $\varepsilon(x-y)$, and denote by $\varphi_{n}$ the harmonic oscillator wave functions. 
For GSE, with weight function $e^{-x^{2}}$ and $N$ odd, we define

$$
\begin{aligned}
S_{N}(x, y) & =\sum_{n=0}^{N-1} \varphi_{n}(x) \varphi_{n}(y)+\sqrt{\frac{N}{2}} \varphi_{N-1}(x) \varepsilon \varphi_{N}(y), \\
I S_{N}(x, y) & =\sum_{n=0}^{N-1} \varepsilon \varphi_{n}(x) \varphi_{n}(y)+\sqrt{\frac{N}{2}} \varepsilon \varphi_{N-1}(x) \varepsilon \varphi_{N}(y), \\
S_{N} D(x, y) & =-\sum_{n=0}^{N-1} \varphi_{n}(x) \varphi_{n}^{\prime}(y)-\sqrt{\frac{N}{2}} \varphi_{N-1}(x) \varphi_{N}(y) .
\end{aligned}
$$

Then

$$
K_{N}(x, y)=\frac{1}{2}\left(\begin{array}{cc}
S_{N}(x, y) & S_{N} D(x, y) \\
I S_{N}(x, y) & S_{N}(y, x)
\end{array}\right) .
$$

For GOE, with weight function $e^{-x^{2} / 2}$ and $N$ even we define $S_{N}(x, y), I S_{N}(x, y)$ and $S_{N} D(x, y)$ by exactly the same formulas and then

$$
K_{N}(x, y)=\left(\begin{array}{cc}
S_{N}(x, y) & S_{N} D(x, y) \\
I S_{N}(x, y)-\varepsilon(x-y) & S_{N}(y, x)
\end{array}\right) .
$$

Despite their apparent similarity the kernels for GSE and GOE have very different properties. First, the functions $\varepsilon \varphi_{n}$, which do not vanish at $\pm \infty$ when $n$ is even, make it appear that the entries of $K_{N}(x, y)$ do not vanish at $\pm \infty$, but they are actually exponentially small for GSE. (See derivation in Section 8 of [10]). Thus the $f$ in (2.1) can be quite general and even grow at $\pm \infty$, and $K_{N} f$ is a trace class operator on $L^{2} \oplus L^{2}$.

This is not the case for GOE. First, the entries do not all vanish at $\pm \infty$; second, the discontinuity in $\varepsilon(x-y)$ prevents $K_{N} f$ from being a trace class operator. (The discussion in Section 9 of [10] skirted these issues.) To take care of these problems we use weighted $L^{2}$ spaces and a generalization of the determinant. Let $\rho$ be any weight function such that $\rho^{-1} \in L^{1}$ and such that all $\varphi_{n} \in L^{2}(\rho) .{ }^{1}$ For any such $\rho$ the matrix $K_{N}$ is a HilbertSchmidt operator on $L^{2}(\rho) \oplus L^{2}\left(\rho^{-1}\right),{ }^{2}$ and this is the space on which $K_{N}$ acts. The diagonal entries of $K_{N}$ are finite rank, hence trace class. Now the definition of determinant extends to Hilbert-Schmidt operator matrices $T$ with trace class diagonal entries by setting $\operatorname{det}(I-T)=\operatorname{det}_{2}(I-T) e^{-\operatorname{tr} T}$, where $\operatorname{tr} T$ denotes the sum of the traces of the diagonal entries of $T$ and $\operatorname{det}_{2}$ is the regularized 2-determinant. ${ }^{3}$ It follows from an identity for 2determinants ([4], p. 169) that for this extended definition we still have the

\footnotetext{
${ }^{1}$ Recall that $L^{2}(\rho)=\left\{h: \int|h(x)|^{2} \rho(x) d x<\infty\right\}$.

2 That $\varepsilon$ is a Hilbert-Schmidt operator from $L^{2}(\rho)$ to $L^{2}\left(\rho^{-1}\right)$ is equivalent to $\rho^{-1} \in L^{1}$. 3 If $T$ is a Hilbert-Schmidt operator with eigenvalues $\mu_{k}$ then $\operatorname{det}_{2}(I-T)=$ $\prod\left(1-\mu_{k}\right) e^{\mu_{k}}$. See [4], Sec. IV.2.
} 
relation

$$
\operatorname{det}\left(I-T_{1}\right)\left(I-T_{2}\right)=\operatorname{det}\left(I-T_{1}\right) \operatorname{det}\left(I-T_{2}\right) .
$$

Using this fact, and defining $\operatorname{det}\left(I-K_{N} f\right)$ in this way, ${ }^{4}$ the discussion of [10] can be carried through for any $f \in L^{\infty}$.

\section{Scaling GSE at the edge.}

Here we follow the notation of Section VII of [9] and denote our scaling transformation by $\tau$, so that

$$
\tau(x)=\sqrt{2 N}+\frac{x}{\sqrt{2} N^{1 / 6}} .
$$

We shall consider only the limit of (2.2). We denote here by $J$ any measurable set which is bounded below and replace $J$ in $(2.2)$ by $\tau(J)$. We shall compute the matrix kernel $K_{G S E}$ such that the limit of the determinant in (2.2) as $N \rightarrow \infty$ is equal to $\operatorname{det}\left(I-K_{G S E} \chi_{J}\right)$.

Observe first that the determinant is unchanged if the kernel $K_{N}(x, y)$ $\chi_{\tau(J)}(y)$ is replaced by

$$
\tau^{\prime} K_{N}(\tau(x), \tau(y)) \chi_{J}(y),
$$

where $\tau^{\prime}=2^{-1 / 2} N^{-1 / 6}$. It is also unchanged if the upper-right entry is multiplied by $\tau^{\prime}$ and the lower-left entry divided by $\tau^{\prime}$. (We shall use the same notation for the modified kernel.) Thus it is a matter of determining the limit, in trace norm, of the entries of the modified matrix kernel.

If we write

$$
\varphi(x)=\left(\frac{N}{2}\right)^{1 / 4} \varphi_{N}(x), \quad \psi(x)=\left(\frac{N}{2}\right)^{1 / 4} \varphi_{N-1}(x),
$$

and set

$$
S_{N}^{0}(x, y)=\sum_{n=0}^{N-1} \varphi_{n}(x) \varphi_{n}(y)
$$

then

$$
S_{N}(x, y)=S_{N}^{0}(x, y)+\psi(x) \varepsilon \varphi(y)
$$

4 The so-defined $\operatorname{det}\left(I-K_{N} f\right)$ is independent of the choice of $\rho$, since eigenfunctions corresponding to nonzero eigenvalues belong to $L^{2}(\rho) \oplus L^{2}\left(\rho^{-1}\right)$ for all permissible $\rho$. In fact it is given by the usual Fredholm expansion, modified for matrix kernels. We prefer the operator definition since it will enable us to establish convergence of the determinants more easily. 
Formula (57) of [9] is in our notation

$$
S_{N}^{0}(x, y)=\int_{0}^{\infty}[\varphi(x+z) \psi(y+z)+\psi(x+z) \varphi(y+z)] d z .
$$

Define $\varphi_{\tau}=\varphi \circ \tau$ and $\psi_{\tau}=\psi \circ \tau$. Then the substitution $z \rightarrow \tau^{\prime} z$ in the integral shows that

$$
\tau^{\prime} S_{N}^{0}(\tau(x), \tau(y))=\tau^{\prime 2} \int_{0}^{\infty}\left[\varphi_{\tau}(x+z) \psi_{\tau}(y+z)+\psi_{\tau}(x+z) \varphi_{\tau}(y+z)\right] d z .
$$

It follows from results on the asymptotics of Hermite polynomials that

$$
\lim _{N \rightarrow \infty} \tau^{\prime} \varphi_{\tau}(x)=\lim _{N \rightarrow \infty} \tau^{\prime} \psi_{\tau}(x)=\frac{1}{\sqrt{2}} A(x)
$$

pointwise, where $A(x)$ denotes the Airy function $\operatorname{Ai}(x)$, and that there are estimates

$$
\tau^{\prime} \varphi_{\tau}(x)=O\left(e^{-x}\right), \quad \tau^{\prime} \psi_{\tau}(x)=O\left(e^{-x}\right)
$$

which hold uniformly in $N$ and for $x$ bounded below. (There is a better bound but this one is good enough for our purposes. See [7], p. 403.) We shall show that this implies that if

$$
K_{\text {Airy }}(x, y)=\int_{0}^{\infty} A(x+z) A(y+z) d z,
$$

then

$$
\tau^{\prime} S_{N}^{0}(\tau(x), \tau(y)) \rightarrow K_{\text {Airy }}(x, y)
$$

in trace norm on any space $L^{2}(s, \infty)$.

To show that

$$
{\tau^{\prime}}^{2} \int_{0}^{\infty} \varphi_{\tau}(x+z) \psi_{\tau}(y+z) d z \rightarrow \frac{1}{2} K_{\mathrm{Airy}}(x, y),
$$

(the other half of $\tau^{\prime} S_{N}^{0}(\tau(x), \tau(y))$ is treated similarly) we write the difference as

$$
\begin{aligned}
\int_{0}^{\infty}\left[\tau^{\prime} \varphi_{\tau}(x+z)-\right. & \left.2^{-1 / 2} A(x+z)\right] \tau^{\prime} \psi_{\tau}(y+z) d z \\
& +\int_{0}^{\infty} 2^{-1 / 2} A(x+z)\left[\tau^{\prime} \psi_{\tau}(y+z)-2^{-1 / 2} A(y+z)\right] d z
\end{aligned}
$$

Each summand is an integral over $z$ of rank one kernels, and the trace norm of an integral is at most the integral of the trace norms. Thus the trace norm of the first summand is at most

$$
\int_{0}^{\infty}\left\|\tau^{\prime} \varphi_{\tau}(x+z)-2^{-1 / 2} A(x+z)\right\|_{2}\left\|\tau^{\prime} \psi_{\tau}(y+z)\right\|_{2} d z
$$


where the first $L^{2}$ norm is taken with respect to the $x$ variable and the second with respect to the $y$ variable. It follows from (3.6) that the second norm is $O\left(e^{-z}\right)$ uniformly in $N$, and from (3.6) and (3.5) that the first norm is also $O\left(e^{-z}\right)$ uniformly in $N$ and tends pointwise to zero. Thus the integral has limit zero. The same argument applies to the other integral.

Thus $S_{N}^{0}$ scales to $K_{\text {Airy }}$ and it remains to consider $\tau^{\prime} \psi_{\tau}(x)(\varepsilon \varphi)_{\tau}(y)$, the last summand in $\tau^{\prime} S_{N}(\tau(x), \tau(y))$. Of course $\tau^{\prime} \psi_{\tau}(x) \rightarrow \frac{1}{\sqrt{2}} A(x)$ in trace norm. Define

$$
\begin{gathered}
c_{\varphi}=\frac{1}{2} \int_{-\infty}^{\infty} \varphi(x) d x, \quad c_{\psi}=\frac{1}{2} \int_{-\infty}^{\infty} \psi(x) d x \\
\Phi_{\tau}(x)=\int_{x}^{\infty} \tau^{\prime} \phi_{\tau}(z) d z,
\end{gathered}
$$

We have

$$
(\varepsilon \varphi)_{\tau}(y)=c_{\varphi}-\int_{\tau(y)}^{\infty} \varphi(z) d z=-\int_{y}^{\infty} \tau^{\prime} \varphi_{\tau}(z) d z=-\Phi(y) .
$$

(Since $N$ is odd so is $\varphi$, so $c_{\varphi}=0$.) From (3.5) and (3.6) it follows that this converges to

$$
-\frac{1}{\sqrt{2}} \int_{y}^{\infty} A(z) d z
$$

in the norm of any space $L^{2}(s, \infty)$.

Thus we have shown that

$$
\tau^{\prime} S_{N}(\tau(x), \tau(y)) \rightarrow K_{\text {Airy }}(x, y)-\frac{1}{2} A(x) \int_{y}^{\infty} A(z) d z
$$

in the trace norm of operators on any space $L^{2}(s, \infty)$.

Next, observe that $S_{N} D(x, y)=-\partial_{y} S_{N}(x, y)$ and so (recall that we multiply the upper-right corner by $\tau^{\prime}$ )

$$
\tau^{\prime 2} S_{N} D(\tau(x), \tau(y))=-\partial_{y} \tau^{\prime} S_{N}(\tau(x), \tau(y)) .
$$

Since the limiting relation we found above for $S_{N}$ holds even after taking $\partial_{y}$ we see that

$$
{\tau^{\prime}}^{2} S_{N} D(\tau(x), \tau(y)) \rightarrow-\partial_{y} K_{\text {Airy }}(x, y)-\frac{1}{2} A(x) A(y)
$$

in trace norm as $N \rightarrow \infty$.

If we recall that we divide the lower-left corner by $\tau^{\prime}$ we see that it remains to find the limit of

$$
I S_{N}(\tau(x), \tau(y))=\varepsilon S_{N}^{0}(\tau(x), \tau(y))+\varepsilon \psi(\tau(x)) \varepsilon \varphi(\tau(y)) .
$$


The last term equals

$$
-\left(c_{\psi}-\Psi_{\tau}(x)\right) \Phi_{\tau}(y) .
$$

If we make the substitution $z \rightarrow \tau^{\prime} z$ in the integral for $\varepsilon S_{N}^{0}(\tau(x), \tau(y))$ it becomes

$$
\int_{0}^{\infty}\left[\left(\varepsilon \varphi_{\tau}\right)(x+z) \tau^{\prime} \psi_{\tau}(y+z)+(\varepsilon \psi)_{\tau}(x+z) \tau^{\prime} \varphi_{\tau}(y+z)\right] d z .
$$

Replacing $\varepsilon \varphi_{\tau}$ and $\varepsilon \psi_{\tau}$ by what they are in terms of $\Phi_{\tau}$ and $\Psi_{\tau}$ gives

$$
-\int_{0}^{\infty}\left[\Phi_{\tau}(x+z) \tau^{\prime} \psi_{\tau}(y+z)+\Psi_{\tau}(x+z) \tau^{\prime} \varphi_{\tau}(y+z)\right] d z+c_{\psi} \Phi_{\tau}(y) .
$$

Putting these together we obtain

$$
\begin{array}{r}
I S_{N}(\tau(x), \tau(y))=-\int_{0}^{\infty}\left[\Phi_{\tau}(x+z) \tau^{\prime} \psi_{\tau}(y+z)+\Psi_{\tau}(x+z) \tau^{\prime} \varphi_{\tau}(y+z)\right] \\
d z+\Psi_{\tau}(x) \Phi_{\tau}(y) .
\end{array}
$$

Now we use (3.5) and (3.6) as before, and deduce that

$$
I S_{N}(\tau(x), \tau(y)) \rightarrow-\int_{x}^{\infty} K_{A}(z, y) d z+\frac{1}{2} \int_{x}^{\infty} A(z) d z \cdot \int_{y}^{\infty} A(z) d z
$$

in trace norm.

Thus if we set

$$
\begin{gathered}
S(x, y)=K_{\text {Airy }}(x, y)-\frac{1}{2} A(x) \int_{y}^{\infty} A(z) d z \\
S D(x, y)=-\partial_{y} K_{\text {Airy }}(x, y)-\frac{1}{2} A(x) A(y), \\
I S(x, y)=-\int_{x}^{\infty} K_{A}(z, y) d z+\frac{1}{2} \int_{x}^{\infty} A(z) d z \cdot \int_{y}^{\infty} A(z) d z,
\end{gathered}
$$

then we have shown that for GSE

$$
\tau^{\prime} K_{N}(\tau(x), \tau(y)) \rightarrow K_{\mathrm{GSE}}(x, y):=\frac{1}{2}\left(\begin{array}{cc}
S(x, y) & S D(x, y) \\
I S(x, y) & S(y, x)
\end{array}\right)
$$

in trace norm as $N \rightarrow \infty$.

In particular, the probability that no eigenvalue lies in $\tau(J)$ tends to

$$
\sqrt{\operatorname{det}\left(I-K_{\mathrm{GSE}} \chi_{J}\right)}
$$

as $N \rightarrow \infty$. 


\section{Scaling GOE at the edge.}

Before computing limits let us see what the norm of a rank one kernel $u(x) v(y)$ is when thought of as taking a space $L^{2}\left(\rho_{1}\right)$ to a space $L^{2}\left(\rho_{2}\right)$. The operator, denoted by $u \otimes v$, takes a function $h \in L^{2}\left(\rho_{1}\right)$ to $u(v, h)$, and so its norm is the $L^{2}\left(\rho_{2}\right)$ norm of $u$ times the norm of $v$ in the space dual to $L^{2}\left(\rho_{1}\right)$, which is $L^{2}\left(\rho_{1}^{-1}\right)$. Thus

$$
\|u \otimes v\|=\|u\|_{L^{2}\left(\rho_{2}\right)}\|v\|_{L^{2}\left(\rho_{1}^{-1}\right)} .
$$

We determine the limit of the scaled kernel

$$
\tau^{\prime} K_{N}(\tau(x), \tau(y))
$$

where $K_{N}$ is now given by (2.4). The space on which this acts is $L^{2}(\rho) \oplus$ $L^{2}\left(\rho^{-1}\right)$ with any fixed permissible $\rho$ which we now assume has at most polynomial growth at $+\infty .{ }^{5}$ As before, we multiply the upper-right corner by $\tau^{\prime}$ and divide the lower-left corner by $\tau^{\prime}$, but do not change notation.

For $\tau^{\prime} S_{N}^{0}(\tau(x), \tau(y))$, the main part of $\tau^{\prime} S_{N}(\tau(x), \tau(y))$, consider the analogue of (3.7). Since this kernel takes $L^{2}(\rho)$ to itself (4.9) gives for the analogue here

$$
\int_{0}^{\infty}\left\|\tau^{\prime} \varphi_{\tau}(x+z)-2^{-1 / 2} A(x+z)\right\|_{L^{2}(\rho)}\left\|\tau^{\prime} \psi_{\tau}(y+z)\right\|_{L^{2}\left(\rho^{-1}\right)} d z
$$

The second factor is $O\left(e^{-z}\right)$ uniformly in $N$ since $\tau^{\prime} \psi_{\tau}(y+z)=O\left(e^{-z}\right)$ and $\rho^{-1} \in L^{1}$. The first factor is at most the square root of

$$
\int_{s}^{\infty}\left|\tau^{\prime} \varphi_{\tau}(x+z)-2^{-1 / 2} A(x+z)\right|^{2} \rho(x) d x
$$

for some $s$. Using (3.5) and (3.6) and the fact that $\rho$ has at most polynomial growth we see that this is uniformly $O\left(e^{-z / 2}\right)$ and converges to zero pointwise. Thus (4.10) has limit zero.

The other integral arising in the aymptotics of $\tau^{\prime} S_{N}(\tau(x), \tau(y))$ is similar, and we deduce that

$$
\tau^{\prime} S_{N}^{0}(\tau(x), \tau(y)) \rightarrow K_{\text {Airy }}(x, y)
$$

in trace norm, as an operator on $L^{2}(\rho)$. It remains to consider $\tau^{\prime} \psi_{\tau}(x)(\varepsilon \varphi)_{\tau}(y)$. First, $\tau^{\prime} \psi_{\tau}(x) \rightarrow \frac{1}{\sqrt{2}} A(x)$ in the space $L^{2}(\rho)$, again by (3.5) and (3.6) and the fact that $\rho$ has at most polynomial growth. Now

$$
(\varepsilon \varphi)_{\tau}(y)=c_{\varphi}-\Phi(y)
$$

\footnotetext{
5 All functions are thought of as defined on some interval $(s, \infty)$.
} 
and since $N$ is even for GOE so is $\varphi$ and $c_{\varphi} \neq 0$. In fact $c_{\varphi} \rightarrow \frac{1}{\sqrt{2}}$ as $N \rightarrow \infty$ and so we see that

$$
(\varepsilon \varphi)_{\tau}(y) \rightarrow \frac{1}{\sqrt{2}}-\frac{1}{\sqrt{2}} \int_{y}^{\infty} A(z) d z
$$

uniformly, and so in the space $L^{2}\left(\rho^{-1}\right)$. Thus,

$$
\tau^{\prime} S_{N}(\tau(x), \tau(y)) \rightarrow K_{\text {Airy }}(x, y)+\frac{1}{2} A(x)\left(1-\int_{y}^{\infty} A(z) d z\right)
$$

in trace norm as an operator on $L^{2}(\rho)$.

Consider now the upper-right corner of the modified matrix,

$$
{\tau^{\prime}}^{2} S_{N} D(\tau(x), \tau(y))=-\partial_{y} \tau^{\prime} S_{N}(\tau(x), \tau(y))-{\tau^{\prime}}^{2} \psi_{\tau}(x) \varphi_{\tau}(y),
$$

whose limit we want to compute as an operator from $L^{2}\left(\rho^{-1}\right)$ to $L^{2}(\rho)$. In the application of (4.9) both norms are that of $L^{2}(\rho)$. In the verification that

$$
-\partial_{y} \tau^{\prime} S_{N}^{0}(\tau(x), \tau(y)) \rightarrow-\partial_{y} K_{\text {Airy }}(x, y)
$$

we obtain an integral of the form (4.10) where the second factor in the integrand is replaced by

$$
\left\|\tau^{\prime} \psi_{\tau}^{\prime}(y+z)\right\|_{L^{2}(\rho)} .
$$

This is bounded uniformly in $N$ and $z$ because (3.6) holds also even after taking derivatives. The first integrand is estimated as before, so the integral tends to zero in trace norm.

We also have $\tau^{\prime} \psi_{\tau}(x) \rightarrow \frac{1}{\sqrt{2}} A(x)$ and $\tau^{\prime} \varphi_{\tau}(y) \rightarrow \frac{1}{\sqrt{2}} A(y)$ in $L^{2}(\rho)$, and so we find that

$$
{\tau^{\prime}}^{2} S_{N} D(\tau(x), \tau(y)) \rightarrow-\partial K_{\text {Airy }}(x, y)-\frac{1}{2} A(x) A(y)
$$

in trace norm as before, but now as operators from $L^{2}\left(\rho^{-1}\right)$ to $L^{2}(\rho)$.

For the lower-left corner, an operator now from $L^{2}(\rho)$ to $L^{2}\left(\rho^{-1}\right)$ so in (4.9) both norms are $L^{2}\left(\rho^{-1}\right)$ norms, we have as before

$$
I S_{N}(\tau(x), \tau(y))=\varepsilon S_{N}^{0}(\tau(x), \tau(y))+\varepsilon \psi(\tau(x)) \varepsilon \varphi(\tau(y)) .
$$

The last term equals

$$
-\Psi_{\tau}(x)\left(c_{\varphi}-\Phi_{\tau}(y)\right)
$$

Replacing $\varepsilon \varphi_{\tau}$ and $\varepsilon \psi_{\tau}$ in (3.8) by what they are in terms of $\Phi_{\tau}$ and $\Psi_{\tau}$ now gives

$$
-\int_{0}^{\infty}\left[\Phi_{\tau}(x+z) \tau^{\prime} \psi_{\tau}(y+z)+\Psi_{\tau}(x+z) \tau^{\prime} \varphi_{\tau}(y+z)\right] d z+c_{\varphi} \Psi_{\tau}(y)
$$


so we obtain now the identity

$$
\begin{aligned}
I S_{N}(\tau(x), \tau(y))= & -\int_{0}^{\infty}\left[\Phi_{\tau}(x+z) \tau^{\prime} \psi_{\tau}(y+z)+\Psi_{\tau}(x+z) \tau^{\prime} \varphi_{\tau}(y+z)\right] d z \\
& +c_{\varphi} \Psi_{\tau}(y)-\Psi_{\tau}(x)\left(c_{\varphi}-\Phi_{\tau}(y)\right) .
\end{aligned}
$$

Now we deduce that

$$
\begin{array}{r}
I S_{N}(\tau(x), \tau(y)) \rightarrow-\int_{x}^{\infty} K_{A}(z, y) d z+\frac{1}{2}\left(\int_{y}^{x} A(z) d z+\int_{x}^{\infty} A(z) d z\right. \\
\left.\cdot \int_{y}^{\infty} A(z) d z\right)
\end{array}
$$

as trace class operators from $L^{2}(\rho)$ to $L^{2}\left(\rho^{-1}\right)$.

Finally, $\varepsilon(\tau(x)-\tau(y))=\varepsilon(x-y)$, so this is unchanged.

Thus if we set

$$
\begin{gathered}
S(x, y)=K_{\text {Airy }}(x, y)+\frac{1}{2} A(x)\left(1-\int_{y}^{\infty} A(z) d z\right), \\
S D(x, y)=-\partial_{y} K_{\text {Airy }}(x, y)-\frac{1}{2} A(x) A(y), \\
I S(x, y)=-\int_{x}^{\infty} K_{A}(z, y) d z+\frac{1}{2}\left(\int_{y}^{x} A(z) d z+\int_{x}^{\infty} A(z) d z \cdot \int_{y}^{\infty} A(z) d z\right),
\end{gathered}
$$

then we have shown that for GOE

$$
\tau^{\prime} K_{N}(\tau(x), \tau(y)) \rightarrow K_{\mathrm{GOE}}(x, y):=\left(\begin{array}{cc}
S(x, y) & S D(x, y) \\
I S(x, y)-\varepsilon(x-y) & S(y, x)
\end{array}\right)
$$

as $N \rightarrow \infty$ in the sense that the kernels converge in the Hilbert-Schmidt norm of operators on $L^{2}(\rho) \oplus L^{2}\left(\rho^{-1}\right)$ and the diagonals converge in trace norm. (In fact all entries converge in trace norm, except for the fixed Hilbert-Schmidt operator $\varepsilon$.)

In particular, it follows from the continuity of the 2-determinant in Hilbert-Schmidt norm and the trace in trace norm that the probability that no eigenvalue lies in $\tau(J)$ tends to

$$
\sqrt{\operatorname{det}\left(I-K_{\mathrm{GOE}} \chi_{J}\right)}
$$

as $N \rightarrow \infty$.

\section{Acknowledgments.}

This work was supported by National Science Foundation under grants DMS-0304414 (first author) and DMS-0243982 (second author). 


\section{BIBLIOGRAPHY}

[1] P.L. Ferrari, Polynuclear growth on a flat substrate and edge scaling of GOE eigenvalues, Commun. Math. Phys., 252 (2004), 77-109.

[2] P.J. Forrester, T. Nagao and G. Honner, Correlations for the orthogonalunitary and symplectic-unitary transitions at the soft and hard edges, Nucl. Phys., B 553 (1999), 601-643.

[3] J. BAIK and E.M. RAIns, Symmetrized random permutations, in Random Matrix Models and Their Applications, eds. P.M. Bleher and A.R. Its, Cambridge Univ. Press, (2001), 1-19.

[4] I.C. Gohberg and M.G. Krein, Introduction to the Theory of Linear Nonselfadjoint Operators, Transl. Math. Monogr., 35, Providence, RI: Amer. Math. Soc., 1969.

[5] K. Johansson, Toeplitz determinants, random growth and determinantal processes, in Proc. of the International Congress of Mathematicians, Vol. III, Higher Education Press, 2002, 53-52.

[6] M.L. Menta, Random Matrices, London: Academic Press, 1991.

[7] F.W.J. Olver, Asymptotics and Special Functions, New York: Academic Press, 1974.

[8] M. PrÄHOFER and H. Spohn, Universal distributions for growth processes in $1+1$ dimensions and random matrices, Phys. Rev. Letts., 84 (2000), 4882-4885.

[9] C.A. Tracy and H. Widom, On orthogonal and symplectic matrix ensembles, Commun. Math. Phys., 177 (1996) 727-754.

[10] C.A. TrACY and H. Widom, Correlation functions, cluster functions and spacing distributions for random matrices, J. Stat. Phys., 92 (1998), 809-835.

[11] C.A. TRACY and H. Widom, Distribution functions for largest eigenvalues and their applications, Proc. of the International Congress of Mathematicians, Vol. I, Higher Education Press, (2002), 587-596.

\author{
Craig A. TRACY, \\ University of California \\ Department of mathematics \\ Davis, CA 95616 (USA) \\ tracy@math.ucdavis.edu \\ Harold WIDOM, \\ University of California \\ Department of mathematics \\ Santa Cruz, CA 95064 (USA) \\ widom@math.ucsc.edu
}


